\title{
Quasi-Cauchy quotients and means
}

\section{JANUSZ MATKOWSKI}

This paper is dedicated to Professor Ludwig Reich on the occasion of his 80th birthday.

Abstract. Let $I \subset \mathbb{R}$ be an interval that is closed under addition, and $k \in \mathbb{N}, k \geq 2$. For a function $f: I \rightarrow(0, \infty)$ such that $F(x):=\frac{f(k x)}{k f(x)}$ is invertible in $I$, the $k$-variable function $M_{f}: I^{k} \rightarrow I$,

$$
M_{f}\left(x_{1}, \ldots, x_{k}\right):=F^{-1}\left(\frac{f\left(x_{1}+\cdots+x_{k}\right)}{f\left(x_{1}\right)+\cdots+f\left(x_{k}\right)}\right),
$$

is a premean in $I$, and it is referred to as a quasi Cauchy quotient of the additive type of generator $f$. Three classes of means of this type generated by the exponential, logarithmic, and power functions, are examined. The suitable quasi Cauchy quotients of the exponential types (for continuous additive, logarithmic, and power functions) are considered. When $I$ is closed under multiplication, the quasi Cauchy quotient means of logarithmic and multiplicative type are studied. The equalities of premeans within each of these classes are discussed and some open problems are proposed.

Mathematics Subject Classification. Primary 26E60, 39B22; Secondary $39 B 12$.

Keywords. Means, Premeans, Quasi-Cauchy quotient, Functional equation, Iteration.

\section{Introduction}

Let $I$ be a real interval that is closed under addition, and $k \geq 2$ be a fixed positive integer. For a function $f: I \rightarrow(0, \infty)$, the $k$-variable function

$$
I^{k} \ni\left(x_{1}, \ldots, x_{k}\right) \longmapsto \frac{f\left(x_{1}+\cdots+x_{k}\right)}{f\left(x_{1}\right)+\cdots+f\left(x_{k}\right)},
$$

called a Cauchy quotient of the additive type (generated by $f$ ), arises when we divide the left-hand side by the right-hand side of the Cauchy functional equation for the additive function $f\left(x_{1}+\cdots+x_{k}\right)=f\left(x_{1}\right)+\cdots+f\left(x_{k}\right)$, 
written in the customary way. The restriction of this function to the main diagonal of $I^{k}$ leads to the single variable function $F(x):=\frac{f(k x)}{k f(x)}$. Theorem 1 in Sect. 3 gives general conditions under which $F$ is invertible, and the $k$ variable function $M_{f}: I^{k} \rightarrow \mathbb{R}$,

$$
M_{f}\left(x_{1}, \ldots, x_{k}\right):=F^{-1}\left(\frac{f\left(x_{1}+\cdots+x_{k}\right)}{f\left(x_{1}\right)+\cdots+f\left(x_{k}\right)}\right),
$$

is a premean or a mean, which is referred to as a quasi-Cauchy quotient of additive-type premean or mean. Of course, additive functions of the form $f(x)=p x$ are useless here. But it is natural to ask what kind of means one can get if the generating function $f$ belongs to one of the three remaining elementary classes of functions: exponential, logarithmic and multiplicative, characterized by the respective Cauchy functional equation. Answering this question we show that the one-parameter family of exponential functions $f(x)=p^{x}$ generates a new class of means (Proposition 1); the logarithmic functions $\log _{p}$ lead to a single premean that is not a mean in any subinterval (Proposition 2); and in the case of power functions, the family of premeans is empty (Remark 8). In the context of Proposition 1, a mean that is invariant with respect to some mean-type mapping appears (Corollary 1).

Since $F$ is invertible iff so is the function $\frac{1}{F}$, and

$$
\left(\frac{1}{F}\right)^{-1}\left(\frac{f\left(x_{1}\right)+\cdots+f\left(x_{k}\right)}{f\left(x_{1}+\cdots+x_{k}\right)}\right)=F^{-1}\left(\frac{f\left(x_{1}+\cdots+x_{k}\right)}{f\left(x_{1}\right)+\cdots+f\left(x_{k}\right)}\right),
$$

there is no need to consider the quasi-Cauchy quotients separately if the roles of nominator and denominator are reversed (Sect. 2, Remarks 3 and 4, concerning also the remaining sections).

In Sect. 4, Theorem 2 gives conditions under which the quasi-Cauchy quotient of exponential-type, i.e.the function

$$
M_{f}\left(x_{1}, \ldots, x_{k}\right)=F^{-1}\left(\frac{f\left(x_{1}+\cdots+x_{k}\right)}{f\left(x_{1}\right) \cdots f\left(x_{k}\right)}\right)
$$

with $F(x)=\frac{f(k x)}{[f(x)]^{k}}$, is a premean or a mean. For the continuous additive functions $f(x)=p x(p>0)$ one gets a $k$-variable mean (independent of $p$ ) which in the case $k=2$ coincides with the harmonic mean (Proposition 3). These means, called Beta-type means, $\mathcal{B}_{k}:(0, \infty)^{k} \rightarrow(0, \infty)$,

$$
\mathcal{B}_{k}\left(x_{1}, \ldots, x_{k}\right)=\left(\frac{k x_{1} \cdots x_{k}}{x_{1}+\cdots+x_{k}}\right)^{\frac{1}{k-1}},
$$

have appeared recently in [4], where the question: when the direct exponential Cauchy quotient is a premean or a mean was considered. For the continuous logarithmic functions $f=\log _{p}$ the respective mean does not depend on $p$, but, only in the case $k=2$, the explicit formula is given (Proposition 4). In the remaining case, if $f$ is a continuous multiplicative function in $(0, \infty)$, 
i.e. $f(x)=x^{p}$ for some $p \in \mathbb{R}, p \neq 0$, then, independently of $p$, the quasiCauchy quotient mean generated by $f$ is the Beta-type mean (Proposition 5).

Let $I$ be a real interval that is closed under addition. In Sect. 5, Theorem 3 provides conditions under which the quasi-Cauchy quotient of logarithmic type

$$
M_{f}\left(x_{1}, \ldots, x_{k}\right):=F^{-1}\left(\frac{f\left(x_{1} \cdots x_{k}\right)}{f\left(x_{1}\right)+\cdots+f\left(x_{k}\right)}\right),
$$

with $F(x):=\frac{f\left(x^{k}\right)}{k f(x)}$, is a premean or a mean. In this case the continuous additive functions $f(x)=p x(p \neq 0)$ generate Beta-type means (Proposition 6), obtained also in the previous section. The exponential functions produce a new class of means described for arbitrary $k \geq 3$ by an implicit equation, and effectively for $k=2$ (Proposition 7). It is interesting that power functions generate an extended family of Beta-type means (Proposition 8).

In Sect. 6, Theorem 4 gives conditions guaranteeing that the quasi-Cauchy quotient of multiplicative type

$$
M_{f}\left(x_{1}, \ldots, x_{k}\right):=F^{-1}\left(\frac{f\left(x_{1} \cdots x_{k}\right)}{f\left(x_{1}\right) \cdots \cdot f\left(x_{k}\right)}\right)
$$

with $F(x):=\frac{f\left(x^{k}\right)}{[f(x)]^{k}}$, is a premean or a mean. Applying Theorem 4 for the exponential functions $f(x)=p^{x}$ one gets a new family of means given, in general, by an implicit formula, which in case $k=2$ reduces to the (translated) geometric mean $M_{f}(x, y)=1+\sqrt{(x-1)(y-1)}$ for all $x, y>1$ (Proposition 9). Proposition 10 describes the means generated by Theorem 4 and the logarithmic functions. Moreover it turns out that in this case the additive continuous functions do not produce any means (Remark 18).

The Beta-type mean $\mathcal{B}_{k}$ plays an important role here, as it appears naturally in each of the sections and helps to describe some of the obtained classes of means.

After each theorem, we pose a problem concerning the equality of the introduced mean. We give only a partial solution of Problem 4, concerning the equality of two multiplicative-type quasi-Cauchy premeans. The suitable functional equations restricted to the diagonal are related to the problem posed by Reich [9].

Let us note that the question when the quasi-Cauchy difference is a mean is considered in [8]. 


\section{Preliminaries}

Let $I \subset \mathbb{R}$ be an interval and $k \in \mathbb{N}, k \geq 2$, be fixed. Recall that a function $M: I^{k} \rightarrow \mathbb{R}$ is called a $k$-variable mean in $I$, if

$$
\min \left(x_{1}, \ldots, x_{k}\right) \leq M\left(x_{1}, \ldots, x_{k}\right) \leq \max \left(x_{1}, \ldots, x_{k}\right), x_{1}, \ldots, x_{k} \in I ;
$$

(see, for instance, [1]). A function $M: I^{k} \rightarrow \mathbb{R}$ is called reflexive if $M(x, \ldots, x)$ $=x$ for all $x \in I$. It is called a $k$-variable premean in $I$, if it is reflexive and $M: I^{k} \rightarrow I$.

A $k$-variable mean or premean in $I$ is called:

strict in the ith variable, if, for all $x_{1}, \ldots, x_{k} \in I$,

$$
M\left(x_{1}, \ldots, x_{i}, \ldots, x_{k}\right)=x_{i} \Longrightarrow x_{1}=x_{2}=\cdots=x_{k},
$$

and it is called strict, if it is strict with respect to each variable; symmetric, if $M\left(x_{\sigma(1)}, \ldots, x_{\sigma(k)}\right)=M\left(x_{1}, \ldots, x_{k}\right)$ for every $\left(x_{1}, \ldots, x_{k}\right) \in I^{k}$ and every permutation $\sigma$ of $\{1, \ldots, k\}$; homogeneous, if $I=(0, \infty)$ and

$$
M\left(t x_{1}, \ldots, t x_{k}\right)=t M\left(x_{1}, \ldots, x_{k}\right), t, x_{1}, \ldots, x_{k}>0 .
$$

Of course, every mean is a premean, but the converse implication is not true. However we have the following

Remark 1. If a function $M: I^{k} \rightarrow \mathbb{R}$ is reflexive and (strictly) increasing in each variable, then it is a (strict) mean; in the sequel it is referred to as a (strictly) increasing $k$-variable mean.

In this connection we have

Remark 2. There is no mean that is decreasing with respect to any variable.

Note the following general method of construction of premeans and means.

Lemma 1. Let $k \in \mathbb{N}, k \geq 2, I \subset \mathbb{R}$ be an interval. For a function $g: I^{k} \rightarrow \mathbb{R}$ define $\gamma: I \rightarrow \mathbb{R}$ by

$$
\gamma(x):=g(x, \ldots, x), \quad x \in I .
$$

Then:

(i) if $g\left(I^{k}\right) \subset \gamma(I)$ and $\gamma$ is invertible, then the function

$$
M:=\gamma^{-1} \circ g
$$

is a well defined $k$-variable premean in $I$;

(ii) if $g$ is continuous and strictly increasing in each variable (strictly decreasing in each variable), then $\gamma$ is continuous strictly increasing (strictly decreasing), and the function $M$ is a strictly increasing $k$-variable mean in $I$ 
(iii) if $g$ is continuous and increasing in each variable (decreasing in each variable) and $\gamma$ is strictly increasing (strictly decreasing), then $M$ is an increasing $k$-variable mean in $I$.

Remark 3. If $g: I^{k} \rightarrow(0, \infty)$ in the previous lemma, then

$$
M=\left(\frac{1}{\gamma}\right)^{-1} \circ\left(\frac{1}{g}\right) .
$$

Remark 4. For a closed under addition interval $I$, a continuous and strictly monotonic function $f: I \rightarrow(0, \infty)$, and $k \in \mathbb{N}$, let

$$
L\left(x_{1}, \ldots, x_{k}\right):=f\left(x_{1}+\cdots+x_{k}\right), \quad R\left(x_{1}, \ldots, x_{k}\right):=f\left(x_{1}\right)+\cdots+f\left(x_{k}\right)
$$

(the left-hand side and the right-hand side of the additivity equation $\left.f\left(x_{1}+\cdots+x_{k}\right)=f\left(x_{1}\right)+\cdots+f\left(x_{k}\right)\right)$, and put

$$
l(x):=L(x, \ldots, x), \quad r(x):=R(x, \ldots, x) .
$$

By the previous remark, if the function $\frac{l}{r}$ is invertible, then,

$$
\begin{aligned}
& \left(\frac{r}{l}\right)^{-1}\left(\frac{f\left(x_{1}\right)+\cdots+f\left(x_{k}\right)}{f\left(x_{1}+\cdots+x_{k}\right)}\right)=\left(\frac{l}{r}\right)^{-1}\left(\frac{f\left(x_{1}+\cdots+x_{k}\right)}{f\left(x_{1}\right)+\cdots+f\left(x_{k}\right)}\right), \\
& x_{1}, \ldots, x_{k} \in I .
\end{aligned}
$$

Similar equalities hold true for the exponential-type, logarithmic type, and multiplicative- (or power)-type Cauchy quotients.

\section{Additive quasi-Cauchy quotient}

Theorem 1. Let $I \subset \mathbb{R}$ be an interval that is closed under addition, i.e. $I+I \subset$ $I ; f: I \rightarrow(0, \infty)$ be a function; $k \in \mathbb{N}, k \geq 2$; and let $F: I \rightarrow(0, \infty)$ be given by

$$
F(x):=\frac{f(k x)}{k f(x)}, \quad x \in I .
$$

Then

(i) if $F$ is one-to-one and

$$
\left\{\frac{f\left(x_{1}+\cdots+x_{k}\right)}{f\left(x_{1}\right)+\cdots+f\left(x_{k}\right)}: x_{1}, \ldots, x_{k} \in I\right\} \subset\{F(x): x \in I\},
$$

then the function $M_{f}: I^{k} \rightarrow(0, \infty)$ defined by

$$
M_{f}\left(x_{1}, \ldots, x_{k}\right):=F^{-1}\left(\frac{f\left(x_{1}+\cdots+x_{k}\right)}{f\left(x_{1}\right)+\cdots+f\left(x_{k}\right)}\right)
$$

is a correctly defined symmetric $k$-variable premean in $I$; 
(ii) if $f$ is continuous and the function

$$
I^{k} \ni\left(x_{1}, \ldots, x_{k}\right) \longmapsto \frac{f\left(x_{1}+\cdots+x_{k}\right)}{f\left(x_{1}\right)+\cdots+f\left(x_{k}\right)}
$$

is strictly increasing (decreasing) in one of the variables, then $F$ is strictly increasing (decreasing) and the function $M_{f}$ defined by (3) is a continuous symmetric strictly increasing $k$-variable mean in $I$.

Proof. (i) By (2) the function $M_{f}$ is well defined and $M_{f}\left(x_{1}, \ldots, x_{k}\right) \in I$ for all $x_{1}, \ldots, x_{k} \in I$. Moreover, in view of (1) and (3), for every $x \in I$ we have

$$
M_{f}(x, \ldots, x):=F^{-1}\left(\frac{f(k x)}{k f(x)}\right)=x,
$$

so $M_{f}$ is reflexive.

To show (ii) assume that the function (4) is strictly increasing in one of the variables. The symmetry of this function implies that it is strictly increasing in each of the variables. Hence, if $x, y \in I, x<y$, then

$F(x)=\frac{f(k x)}{k f(x)}=\frac{f(x+\cdots+x)}{f(x)+\cdots+f(x)} \leq \frac{f(y+\cdots+y)}{f(y)+\cdots+f(y)}=\frac{f(k y)}{k f(y)}=F(y)$,

so $F$ is increasing. Since $F$ is one-to-one, it is strictly increasing. The function $M_{f}$, being the composition of the function (4) and $F^{-1}$, is (strictly) increasing. Since, in view of (i), $M_{f}$ is a premean, it is reflexive.

In the case when the function (4) is strictly decreasing in one of the variables, arguing similarly, we show that $F$ is strictly decreasing. Consequently, the function $M_{f}$, being a composition of two strictly decreasing functions, is increasing. In both cases the continuity of $M_{f}$ is obvious.

Now (ii) follows from Remark 1.

Definition 1. Under the assumptions of Theorem 1 (ii), the function $M_{f}: I^{k} \rightarrow$ $I$ is referred to as an additive-type quasi-Cauchy quotient mean generated by $f$ (or of generator $f$ ).

Remark 5. There is no monotonic function $f:(0, \infty) \rightarrow(0, \infty)$ such that its direct additive-type Cauchy quotient is a premean in $(0, \infty)$.

To see this assume, on the contrary, that for some monotonic function $f:(0, \infty) \rightarrow(0, \infty)$, the function $M:(0, \infty)^{2} \rightarrow(0, \infty), M(x, y)=\frac{f(x+y)}{f(x)+f(y)}$ is a premean. Then $\frac{f(2 x)}{2 f(x)}=x$ for all $x>0$, or, equivalently, $f$ satisfies the iterative functional equation

$$
f(x)=x f\left(\frac{x}{2}\right), \quad x>0 .
$$

Hence, by induction, we get

$$
f(x)=x \frac{x}{2} \frac{x}{2^{2}} \cdots \cdots \frac{x}{2^{n-1}} f\left(\frac{x}{2^{n}}\right)=\frac{x^{n}}{2^{\frac{n(n-1)}{2}}} f\left(\frac{x}{2^{n}}\right), \quad x>0, n \in \mathbb{N} .
$$


If $f$ were bounded in a right vicinity of 0 , then, letting $n \rightarrow \infty$, we would get $f(x)=0$ for all $x>0$.

Thus $\lim _{x \rightarrow 0} f(x)=\infty$. Consequently, $f$ must be decreasing and $\lim _{x \rightarrow \infty} f(x)$ is a finite nonnegative real number. It follows that $\frac{f(2 x)}{2 f(x)} \leq$ $\frac{f(x)}{2 f(x)}=\frac{1}{2}<x$ for all $x>0$ is invalid, so $M$ is not reflexive.

Since a positive continuous solution of this iterative equation depends on an arbitrary function (see [6]), the condition of monotonicity of $f$ in Remark 5 is essential.

Problem 1. Let $f: I \rightarrow(0, \infty)$ and $g: I \rightarrow(0, \infty)$ satisfy the conditions of Theorem 1. Determine conditions under which the quasi-Cauchy quotient premeans $M_{f}$ and $M_{g}$ are equal.

Remark 6. Put $G(x):=\frac{g(k x)}{k g(x)}$ and $\varphi:=G \circ F^{-1}$. The equality $M_{f}=M_{g}$ leads to the functional equation

$$
\varphi\left(\frac{f\left(x_{1}+\cdots+x_{k}\right)}{f\left(x_{1}\right)+\cdots+f\left(x_{k}\right)}\right)=\frac{g\left(x_{1}+\cdots+x_{k}\right)}{g\left(x_{1}\right)+\cdots+g\left(x_{k}\right)}, \quad x_{1}, \ldots, x_{k} \in I,
$$

which for $k=2$ reduces to

$$
\varphi\left(\frac{f(x+y)}{f(x)+f(y)}\right)=\frac{g(x+y)}{g(x)+g(y)}, \quad x, y \in I,
$$

which is a special case of a more general functional equation

$$
\varphi(h(x, y))=\frac{g(x+y)}{g(x)+g(y)}, \quad x, y \in I,
$$

in which $\varphi, h$ and $g$ are unknown.

Part 1: Additive quasi-Cauchy quotient for exponential functions

Applying Theorem 1 for the exponential function of the form $f(x)=p^{x}$ we get

Proposition 1. Let $k \in \mathbb{N}, k \geq 2$, be fixed. For every $p>0, p \neq 1$, the function $M_{p}: \mathbb{R}^{k} \rightarrow \mathbb{R}$ given by

$$
M_{p}\left(x_{1}, \ldots, x_{k}\right)=\frac{1}{k-1} \log _{p} \frac{k p^{x_{1}+\cdots+x_{k}}}{p^{x_{1}}+\cdots+p^{x_{k}}}
$$

is an additive type quasi-Cauchy quotient mean generated by the exponential function $f(x)=p^{x},(x \in \mathbb{R})$. 
Moreover, for all $x_{1}, \ldots, x_{k} \in \mathbb{R}$,

$$
\begin{aligned}
& \lim _{p \rightarrow 1} M_{p}\left(x_{1}, \ldots, x_{k}\right)=\frac{x_{1}+\cdots+x_{k}}{k} ; \\
& \lim _{p \rightarrow 0} M_{p}\left(x_{1}, \ldots, x_{k}\right) \\
= & \frac{\operatorname{card}\left\{j: x_{j}=\min \left(x_{1}, \ldots, x_{k}\right)\right\}-1}{k-1} \min \left(x_{1}, \ldots, x_{k}\right)+\frac{1}{k-1} \sum_{\left\{j: x_{j} \neq\right.} \\
& \left.\min \left(x_{1}, \ldots, x_{k}\right)\right\} x_{j} ; \\
& \lim _{p \rightarrow \infty} M_{p}\left(x_{1}, \ldots, x_{k}\right) \\
= & \frac{\operatorname{card}\left\{j: x_{j}=\max \left(x_{1}, \ldots, x_{k}\right)\right\}-1}{k-1} \max \left(x_{1}, \ldots, x_{k}\right)+\frac{1}{k-1} \sum_{\left\{j: x_{j} \neq\right.} \\
& \left.\max \left(x_{1}, \ldots, x_{k}\right)\right\} x_{j} ;
\end{aligned}
$$

and setting

$$
M_{1}:=\lim _{p \rightarrow 1} M_{p}, \quad M_{0}:=\lim _{p \rightarrow 0} M_{p}, \quad M_{\infty}:=\lim _{p \rightarrow \infty} M_{p}
$$

(pointwise limits), yields a family of symmetric means $\left\{M_{q}: q \in[0, \infty]\right\}$ such that for every $x_{1}, \ldots, x_{k} \in \mathbb{R}$, the function

$$
[0, \infty] \ni q \longmapsto M_{q}\left(x_{1}, \ldots, x_{k}\right) \text { is continuous. }
$$

Proof. Take $k \in \mathbb{N}, k \geq 2, I=\mathbb{R}, p>0, p \neq 1$, and the function $f(x)=p^{x}$. By (11) we have

$$
F(x)=\frac{p^{k x}}{k p^{x}}=\frac{p^{(k-1) x}}{k}, \quad x \in \mathbb{R} .
$$

The function $F$ maps $\mathbb{R}$ onto $(0, \infty)$, it is strictly increasing if $p>1$ and strictly decreasing if $0<p<1$; so $F$ satisfies all the conditions of Theorem 1(ii). To show that the function

$$
\mathbb{R}^{k} \ni\left(x_{1}, \ldots, x_{k}\right) \longmapsto \frac{p^{x_{1}+\cdots+x_{k}}}{p^{x_{1}}+\cdots+p^{x_{k}}}
$$

is strictly monotonic with respect to the first variable, let us fix arbitrarily $x_{2}, \ldots, x_{k} \in \mathbb{R}$, and define $\varphi: \mathbb{R} \rightarrow \mathbb{R}$ by

$$
\varphi(t):=M_{r}\left(t, x_{2}, \ldots, x_{k}\right)=\frac{a r^{t}}{r^{t}+b},
$$

where $a:=r^{x_{2}+\cdots+x_{k}}$ and $b:=r^{x_{2}}+\cdots+r^{x_{k}}$ are some positive real numbers. The function $\varphi$, being the composition of the strictly increasing homographic function $(0, \infty) \ni u \longmapsto \frac{a u}{u+m}$ and the exponential function $\mathbb{R} \ni u \longmapsto p^{u}$, is strictly increasing if $p>1$, and strictly decreasing if $0<p<1$. It follows that the function $M_{p}$ is strictly monotonic with respect to the first variable. 
The symmetry of $M_{p}$ implies that it is strictly monotonic with respect to each variable, and, consequently, all the assumptions of Theorem 1 (ii) are satisfied.

Since

$$
F^{-1}(t)=\frac{1}{k-1} \log _{p} k t, \quad t \in(0, \infty),
$$

applying Theorem 1(ii), we get

$$
M_{p}\left(x_{1}, \ldots, x_{k}\right)=\frac{1}{k-1} \log _{p} \frac{k p^{x_{1}+\cdots+x_{k}}}{p^{x_{1}}+\cdots+p^{x_{k}}}, \quad x_{1}, \ldots, x_{k} \in \mathbb{R} .
$$

Since

$$
M_{p}\left(x_{1}, \ldots, x_{k}\right)=\frac{1}{k-1} \frac{\ln \frac{k e^{\left(x_{1}+\cdots+x_{k}\right) \ln p}}{e^{x_{1} \ln p+\cdots+e^{x_{k} \ln p}}}}{\ln p}, \quad x_{1}, \ldots, x_{k} \in \mathbb{R},
$$

and, after an easy calculation,

$$
\begin{aligned}
& \frac{\frac{\partial}{\partial p}\left(\ln \frac{k e^{\left(x_{1}+\cdots+x_{k}\right) \ln p}}{e^{x_{1} \ln p}+\cdots+e^{x_{k} \ln p}}\right)}{\frac{\partial}{\partial p}(\ln p)} \\
= & \frac{\left(x_{1}+\cdots+x_{k}\right)\left(e^{x_{1} \ln p}+\cdots+e^{x_{k} \ln p}\right)-\left(e^{x_{1} \ln p} x_{1}+\cdots+e^{x_{k} \ln p} x_{k}\right)}{e^{x_{1} \ln p}+\cdots+e^{x_{k} \ln p}},
\end{aligned}
$$

by the L'Hospital rule, we obtain

$$
\begin{aligned}
& (k-1) \lim _{p \rightarrow 1} M_{p}\left(x_{1}, \ldots, x_{k}\right) \\
= & \lim _{p \rightarrow 1} \frac{\left(e^{x_{1} \ln p}+\cdots+e^{x_{k} \ln p}\right)\left(x_{1}+\cdots+x_{k}\right)-\left(e^{x_{1} \ln p} x_{1}+\cdots+e^{x_{k} \ln p} x_{k}\right)}{e^{x_{1} \ln p}+\cdots+e^{x_{k} \ln p}} \\
= & \frac{k\left(x_{1}+\cdots+x_{k}\right)-\left(x_{1}+\cdots+x_{k}\right)}{k}=\frac{(k-1)\left(x_{1}+\cdots+x_{k}\right)}{k},
\end{aligned}
$$

whence

$$
M_{1}\left(x_{1}, \ldots, x_{k}\right):=\lim _{p \rightarrow 1} M_{p}\left(x_{1}, \ldots, x_{k}\right)=\frac{x_{1}+\cdots+x_{k}}{k}, \quad x_{1}, \ldots, x_{k} \in \mathbb{R} .
$$

To calculate the limit at 0 , assume first that $x_{1}, \ldots, x_{k} \in \mathbb{R}$ are such that

$$
x_{1}=\min \left(x_{1}, \ldots, x_{k}\right)<\min \left(x_{2}, \ldots, x_{k}\right) .
$$

Making use of the above formula, we have

$$
\begin{aligned}
& \frac{\frac{\partial}{\partial p}\left(\ln \frac{k e^{\left(x_{1}+\cdots+x_{k}\right) \ln p}}{e^{x_{1} \ln p}+\cdots+e^{x_{k} \ln p}}\right)}{\frac{\partial}{\partial p}(\ln p)}=\frac{1}{1+e^{\left(x_{2}-x_{1}\right) \ln p}+\cdots+e^{\left(x_{k}-x_{1}\right) \ln p}} \\
& \cdot\left(\begin{array}{c}
\left(1+e^{\left(x_{2}-x_{1}\right) \ln p}+\cdots+e^{\left(x_{k}-x_{1}\right) \ln p}\right)\left(x_{1}+\cdots+x_{k}\right) \\
-\left(x_{1}+e^{\left(x_{2}-x_{1}\right) \ln p} x_{2}+\cdots+e^{\left(x_{k}-x_{1}\right) \ln p} x_{k}\right)
\end{array}\right) .
\end{aligned}
$$


By the L'Hospital rule, taking into account that the numbers $x_{2}-x_{1}, \ldots, x_{k}-$ $x_{1}$ are positive, and letting $p \rightarrow 0$, we get

$$
(k-1) \lim _{p \rightarrow 0} M_{p}\left(x_{1}, \ldots, x_{k}\right)=\left(x_{1}+\cdots+x_{k}\right)-x_{1},
$$

SO

$$
M_{0}\left(x_{1}, \ldots, x_{k}\right)=\lim _{p \rightarrow 0} M_{p}\left(x_{1}, \ldots, x_{k}\right)=\frac{x_{1}+\cdots+x_{k}-\min \left(x_{1}, \ldots, x_{k}\right)}{k-1} .
$$

Now assume that $x_{1}, \ldots, x_{k} \in \mathbb{R}$ are such that, for some $l \in \mathbb{N}, 1<l<k$,

$$
x_{1}=x_{2}=\cdots=x_{l}=\min \left(x_{1}, \ldots, x_{k}\right)<\min \left(x_{l+1}, \ldots, x_{k}\right) .
$$

In this case we have

$$
\begin{aligned}
& \frac{\frac{\partial}{\partial p}\left(\ln \frac{k e^{\left(x_{1}+\cdots+x_{k}\right) \ln p}}{e^{x_{1} \ln p+\cdots+e^{x_{k} \ln p}}}\right)}{\frac{\partial}{\partial p}(\ln p)}=\frac{1}{l+e^{\left(x_{l+1}-x_{1}\right) \ln p}+\cdots+e^{\left(x_{k}-x_{1}\right) \ln p}} \\
& \cdot\left(\begin{array}{c}
\left(l+e^{\left(x_{l+1}-x_{1}\right) \ln p}+\cdots+e^{\left(x_{k}-x_{1}\right) \ln p}\right)\left(x_{1}+\cdots+x_{k}\right) \\
-\left(x_{1}+\cdots+x_{l}+e^{\left(x_{l+1}-x_{1}\right) \ln p} x_{l+1}+\cdots+e^{\left(x_{k}-x_{1}\right) \ln p} x_{k}\right)
\end{array}\right),
\end{aligned}
$$

and letting $p \rightarrow 0$, we get

$$
\begin{aligned}
& (k-1) \lim _{p \rightarrow 0} M_{p}\left(x_{1}, \ldots, x_{k}\right) \\
= & \frac{l^{2} x_{1}+l\left(x_{l+1}+\cdots+x_{k}\right)-l x_{1}}{l}=(l-1) x_{1}+x_{l+1}+\cdots+x_{k},
\end{aligned}
$$

so, generally,

$$
\begin{aligned}
& M_{0}\left(x_{1}, \ldots, x_{k}\right)=\lim _{p \rightarrow 0} M_{p}\left(x_{1}, \ldots, x_{k}\right) \\
& =\frac{(l-1) \min \left(x_{1}, \ldots, x_{k}\right)+x_{l+1}+\cdots+x_{k}}{k-1} .
\end{aligned}
$$

Now the symmetry of $M_{p}$ implies that for all $x_{1}, \ldots, x_{k} \in \mathbb{R}$,

$$
\begin{aligned}
& \lim _{p \rightarrow 0} M_{p}\left(x_{1}, \ldots, x_{k}\right) \\
= & \frac{\left(\left(\sum_{\left\{j: x_{j}=\min \left(x_{1}, \ldots, x_{k}\right)\right\}} j\right)-1\right) \min \left(x_{1}, \ldots, x_{k}\right)+\sum_{\left\{j: x_{j} \neq \min \left(x_{1}, \ldots, x_{k}\right)\right\}} x_{j}}{k-1}, \\
= & \frac{\operatorname{card}\left\{j: x_{j}=\min \left(x_{1}, \ldots, x_{k}\right)\right\}-1}{k-1} \min \left(x_{1}, \ldots, x_{k}\right) \\
& \quad+\frac{\sum_{\left\{j: x_{j} \neq \min \left(x_{1}, \ldots, x_{k}\right)\right\}} x_{j}}{k-1},
\end{aligned}
$$

and, of course,

$$
M_{0}:=\lim _{p \rightarrow 0} M_{p}
$$


is a symmetric mean.

We omit the analogous reasoning in the calculations of $\lim _{p \rightarrow \infty} M_{p}$.

Remark 7. Proposition 1 implies that for all $k \geq 2$ and $p>0, p \neq 1$,

$$
M_{p}\left(x_{1}, \ldots, x_{k}\right)=\log _{p} \mathcal{B}_{k}\left(p^{x_{1}}, \ldots, p^{x_{k}}\right), \quad x_{1}, \ldots, x_{k} \in \mathbb{R},
$$

so $M_{p}$ is exponentially conjugate to $\mathcal{B}_{k}$, the Beta-type mean defined in the introduction. In the case $k=2$ we get $M_{p}(x, y)=\log _{p} \mathcal{H}\left(p^{x}, p^{y}\right)$, where $\mathcal{H}:(0, \infty)^{2} \rightarrow(0, \infty)$, given by $\mathcal{H}(x, y)=\frac{2 x y}{x+y}$ is the harmonic mean. Thus $M_{p}$ is exponentially conjugate to the harmonic mean.

Corollary 1. Let $k=2$. Then for every $q>0$ the arithmetic mean $A=M_{1}$ is invariant with respect to the mean-type mapping $\left(M_{\frac{1}{q}}, M_{q}\right):(0, \infty)^{2} \rightarrow$ $(0, \infty)^{2}$, i.e.

$$
M_{1} \circ\left(M_{\frac{1}{q}}, M_{q}\right)=M_{1},
$$

which implies that the sequence $\left(\left(M_{\frac{1}{q}}, M_{q}\right)^{n}: n \in \mathbb{N}\right)$ of iterates of $\left(M_{\frac{1}{q}}, M_{q}\right)$ is pointwise convergent in $\mathbb{R}^{2}$ and

$$
\lim _{n \rightarrow \infty}\left(M_{\frac{1}{q}}, M_{q}\right)^{n}=\left(M_{1}, M_{1}\right) .
$$

Proof. Since, after simple calculations, for all $x, y \in \mathbb{R}$,

$$
\begin{aligned}
& M_{1} \circ\left(M_{\frac{1}{q}}, M_{q}\right)(x, y)=\frac{1}{2}\left(\log _{\frac{1}{q}} \frac{2 \frac{1}{q^{x+y}}}{\frac{1}{q^{x}}+\frac{1}{q^{y}}}+\log _{q} \frac{2 q^{x+y}}{q^{x}+q^{y}}\right) \\
& \quad=\frac{x+y}{2}=M_{1}(x, y),
\end{aligned}
$$

the mean $M_{1}$ is $\left(M_{\frac{1}{q}}, M_{q}\right)$-invariant, and the result follows from [7].

\section{Part 2: Additive quasi-Cauchy quotient for logarithmic functions}

Proposition 2. Let $k \in \mathbb{N}, k \geq 2 ; p>0, p \neq 1$, be fixed and let $f=\log _{p}$. Then

(i) for every $p>0, p \neq 1$, the additive type quasi-Cauchy quotient $M_{f}$ : $(1, \infty)^{k} \rightarrow(1, \infty)$ generated by the logarithmic function $f=\log _{p}$ is a premean, it does not depend on $p$, and $M_{f}=M_{\text {ln }}$ where

$$
M_{\ln }\left(x_{1}, \ldots, x_{k}\right):=k^{\frac{\ln \left(x_{1} \cdots \cdots x_{k}\right)}{k\left(\ln \left(x_{1}+\cdots+x_{k}\right)-\ln x_{1} \cdots \cdots x_{k}\right)}}, \quad x_{1}, \ldots, x_{k}>1 ;
$$

(ii) the premean $M_{\mathrm{ln}}$ is not a mean in any of the intervals $(\alpha, \infty)$ where $\alpha \geq 1$. 
Proof. Take $k \in \mathbb{N}, k \geq 2, p>0, p \neq 1$, the interval $I=(1, \infty)$, and the function $f: I \rightarrow \mathbb{R}, f=\log _{p}$. By (1) we have

$$
F(x)=\frac{\log _{p}(k x)}{k \log _{p} x}, \quad x>1 .
$$

Hence, since $\log _{p}=\frac{\ln }{\ln p}$, we have

$$
F(x)=\frac{\ln (k x)}{k \ln (x)}, \quad x>1 .
$$

It is easy to verify that $F$ is decreasing in $(1, \infty)$, and maps the interval $(1, \infty)$ onto $\left(\frac{1}{k}, \infty\right)$.

Since $\log _{p}=\frac{\ln }{\ln p}$, the $k$-variable function (4) has the form

$$
(1, \infty)^{k} \ni\left(x_{1}, \ldots, x_{k}\right) \longmapsto \frac{\log _{p}\left(x_{1}+\cdots+x_{k}\right)}{\log _{p}\left(x_{1} \cdots x_{k}\right)}=\frac{\ln \left(x_{1}+\cdots+x_{k}\right)}{\ln \left(x_{1} \cdots x_{k}\right)} .
$$

For arbitrarily fixed $x_{2}, \ldots, x_{k} \in(1, \infty)$ define $\varphi:(1, \infty) \rightarrow(0, \infty)$, by

$$
\varphi(t):=\frac{\ln (a+t)}{\ln (b t)}, \quad t>1,
$$

where $a:=x_{2}+\cdots+x_{k}$ and $b:=x_{2} \cdots x_{k}$.

Note that

$$
\varphi^{\prime}(t)=\frac{\psi(t)}{[\ln (b t)]^{2} t(a+t)}, \quad t>1,
$$

where

$$
\psi(t):=t \ln (b t)-(a+t) \ln (a+t), \quad t>1 .
$$

Considering this function we conclude that $\varphi$ has exactly one global minimum at a point $c \in(a, b) \subset(1, \infty)$ and, consequently, the function $\varphi:(1, \infty) \rightarrow$ $(0, \infty)$ is strictly decreasing in the interval $(1, c]$ and is strictly increasing in $[c, \infty)$, for some $c \in(1, \infty)$. This property implies that for arbitrarily fixed $x_{1}, \ldots, x_{k} \in(1, \infty)$ one can find $j \in\{1, \ldots, k\}$ such that

$$
\frac{\ln \left(x_{1}+\cdots+x_{k}\right)}{\ln \left(x_{1} \cdots x_{k}\right)} \geq \frac{\ln \left(x_{j}+\cdots+x_{j}\right)}{\ln \left(x_{j} \cdots x_{j}\right)}=F\left(x_{j}\right)>\frac{1}{k},
$$

which proves that condition (2) of Theorem 1 is satisfied. Since

$$
F^{-1}(t)=e^{\frac{\ln k}{k t-1}}, \quad t>\frac{1}{k},
$$

by Theorem 1 , the function 


$$
\begin{aligned}
& M_{f}\left(x_{1}, \ldots, x_{k}\right)=e^{\frac{\ln k}{k \frac{\ln \left(x_{1}+\cdots+x_{k}\right)}{\ln \left(x_{1} \cdots \cdots x_{k}\right)}-1}}=e^{(\ln k) \frac{\ln \left(x_{1} \cdots \cdots x_{k}\right)}{k\left(\ln \left(x_{1}+\cdots+x_{k}\right)-\ln \left(x_{1} \cdots \cdots x_{k}\right)\right)}} \\
& =\left(e^{\ln k}\right)^{\frac{\ln \left(x_{1} \cdots \cdots x_{k}\right)}{k\left(\ln \left(x_{1}+\cdots+x_{k}\right)-\ln \left(x_{1} \cdots \cdots x_{k}\right)\right)}}=k^{\frac{\ln \left(x_{1} \cdots \cdots x_{k}\right)}{k\left(\ln \left(x_{1}+\cdots+x_{k}\right)-\ln \left(x_{1} \cdots \cdots x_{k}\right)\right)}}
\end{aligned}
$$

is a $k$-variable premean in $(1, \infty)$.

Since, for each $j \in\{1, \ldots, k\}$,

$$
\lim _{x_{j} \rightarrow \infty} \frac{\ln \left(x_{1} \cdots x_{k}\right)}{k\left(\ln \left(x_{1}+\cdots+x_{k}\right)-\ln \left(x_{1} \cdots x_{k}\right)\right)}=\frac{1}{k-1}
$$

for all $x_{1}, \ldots, x_{j-1}, x_{j+1}, \ldots, x_{k} \in(1, \infty)$ we have

$$
\lim _{x_{j} \rightarrow \infty} M_{f}\left(x_{1}, \ldots, x_{k}\right)=k^{\frac{1}{k-1}}, \quad x_{1}, \ldots, x_{j-1}, x_{j+1}, \ldots, x_{k}>1 .
$$

Of course, it follows that $M_{f}:=M_{\mathrm{ln}}$ is not a mean in any closed under addition subinterval $J$ of $(1, \infty)$.

Part 3: Additive quasi-Cauchy quotient for power functions

It turns out that

Remark 8. There are no additive-type quasi-Cauchy quotient premeans generated by power functions.

Indeed, take an arbitrary $k \in \mathbb{N}, k \geq 2$, an interval $I \subset(0, \infty)$, closed under addition, and a power function $f(x)=x^{p}$, where $p \in \mathbb{R}, p \neq 0$. Since by (1),

$$
F(x)=\frac{(k x)^{p}}{k x^{p}}=k^{p-1}, \quad x \in I,
$$

the function $F$ is constant, which shows that there does not exist an additivetype quasi-Cauchy quotient mean generated by $f$.

\section{Exponential quasi-Cauchy quotient}

Theorem 2. Let $I \subset \mathbb{R}$ be an interval that is closed under addition, i.e. $I+I \subset$ $I ; f: I \rightarrow(0, \infty)$ be a function; $k \in \mathbb{N}, k \geq 2$; and let $F: I \rightarrow(0, \infty)$ be given by

$$
F(x):=\frac{f(k x)}{[f(x)]^{k}}, \quad x \in I
$$

Then

(i) if $F$ is one-to-one and

$$
\left\{\frac{f\left(x_{1}+\cdots+x_{k}\right)}{\left.f\left(x_{1}\right) \cdots+x_{k}\right)}: x_{1}, \ldots, x_{k} \in I\right\} \subset\{F(x): x \in I\},
$$


then the function $M_{f}: I^{k} \rightarrow(0, \infty)$ defined by

$$
M_{f}\left(x_{1}, \ldots, x_{k}\right):=F^{-1}\left(\frac{f\left(x_{1}+\cdots+x_{k}\right)}{f\left(x_{1}\right) \cdots \cdot f\left(x_{k}\right)}\right)
$$

is a correctly defined symmetric $k$-variable premean in $I$;

(ii) if $f$ is continuous and the function

$$
I^{k} \ni\left(x_{1}, \ldots, x_{k}\right) \longmapsto \frac{f\left(x_{1}+\cdots+x_{k}\right)}{f\left(x_{1}\right) \cdots \cdot f\left(x_{k}\right)}
$$

is strictly increasing (decreasing) in one of the variables, then $F$ is strictly increasing (decreasing) and the function $M_{f}$ defined by ( 7$)$ is a continuous symmetric strictly increasing $k$-variable mean in $I$.

Proof. Analogous as in Theorem 1.

Definition 2. Under the assumptions of Theorem 2 (ii), the function $M: I^{k} \rightarrow$ $I$ is referred to as an exponential-type quasi-Cauchy quotient mean generated by $f$ (or, of generator $f$ ).

Problem 2. Let $f: I \rightarrow(0, \infty)$ and $g: I \rightarrow(0, \infty)$ satisfy the conditions of Theorem 2. Determine conditions under which the exponential-type quasiCauchy quotient premeans $M_{f}$ and $M_{g}$ are equal.

Remark 9. Put $G(x):=\frac{g(k x)}{k g(x)}$ and $\varphi:=G \circ F^{-1}$. Now the equality $M_{f}=M_{g}$ leads to the functional equation

$$
\varphi\left(\frac{f\left(x_{1}+\cdots+x_{k}\right)}{f\left(x_{1}\right) \cdots+f\left(x_{k}\right)}\right)=\frac{g\left(x_{1}+\cdots+x_{k}\right)}{g\left(x_{1}\right) \cdots \cdot g\left(x_{k}\right)}, \quad x_{1}, \ldots, x \in I,
$$

which for $k=2$ reduces to

$$
\varphi\left(\frac{f(x+y)}{f(x) f(y)}\right)=\frac{g(x+y)}{g(x) g(y)}, \quad x, y \in I,
$$

which is a special case of the functional equation

$$
\varphi(h(x, y))=\frac{g(x+y)}{g(x) g(y)}, \quad x, y \in I,
$$

where $\varphi, h$ and $g$ are unknown.

Part 4: Exponential quasi-Cauchy quotient for additive functions

Applying Theorem 2 we obtain

Proposition 3. Let $k \in \mathbb{N}, k \geq 2$, be fixed. For every $p>0$, the exponential-type quasi-Cauchy quotient mean $M_{f}:(0, \infty)^{k} \rightarrow(0, \infty)$ generated by the function $f(x)=p x,(x>0)$, does not depend on $p$, and

$$
M_{f}=\mathcal{B}_{k}
$$


where $\mathcal{B}_{k}$ is the Beta-type mean given by

$$
\mathcal{B}_{k}\left(x_{1}, \ldots, x_{k}\right)=\left(\frac{k x_{1} \cdots x_{k}}{x_{1}+\cdots+x_{k}}\right)^{1 /(k-1)} .
$$

Proof. Take $k \in \mathbb{N}, k \geq 2, I=(0, \infty), p>0$, and the function $f(x)=p x$. By (5) we have

$$
F(x)=\frac{p k x}{(p x)^{k}}=\frac{k}{p^{k-1} x^{k-1}}, \quad x>0,
$$

so the function $F:(0, \infty) \rightarrow(0, \infty)$ is strictly decreasing. Moreover we have

$$
F^{-1}(t)=\left(\frac{k}{p^{k-1}} \frac{1}{t}\right)^{1 /(k-1)}, \quad t>0 .
$$

Since

$$
\frac{\partial}{\partial x_{1}} \frac{p\left(x_{1}+\cdots+x_{k}\right)}{\left(p x_{1}\right) \cdots \cdot\left(p x_{k}\right)}=-\frac{1}{p^{k-1} x_{1}^{2}} \frac{x_{2}+\cdots+x_{k}}{x_{2} \cdots x_{k}}<0,
$$

the symmetric function

$$
(0, \infty)^{k} \ni\left(x_{1}, \ldots x_{k}\right) \longmapsto \frac{p\left(x_{1}+\cdots+x_{k}\right)}{\left(p x_{1}\right) \cdots\left(p x_{k}\right)}
$$

is strictly decreasing in each variable. By Theorem 2(ii) we obtain, for all $x_{1}, \ldots, x_{k}>0$,

$M_{f}\left(x_{1}, \ldots, x_{k}\right)=\left(\frac{k}{p^{k-1}} \frac{\left(p x_{1}\right) \cdots\left(p x_{k}\right)}{p\left(x_{1}+\cdots+x_{k}\right)}\right)^{1 /(k-1)}=\left(\frac{k x_{1} \cdots \cdots x_{k}}{x_{1}+\cdots+x_{k}}\right)^{1 /(k-1)}$.

Remark 10. The mean $M_{f}$ coincides with the Beta-type $k$-variable mean obtained in [4]. For $k=2$, the mean $M$ is the classical harmonic mean.

\section{Part 5: Exponential quasi-Cauchy quotient for logarithmic functions}

Proposition 4. Let $k \in \mathbb{N}, k \geq 2$, be fixed. For every $p>0, p \neq 1$, the function $M:(1, \infty)^{k} \rightarrow(1, \infty)$ given by the implicit equality

$$
\frac{\ln \left(k M\left(x_{1}, \ldots, x_{k}\right)\right)}{\left[\ln M\left(x_{1}, \ldots, x_{k}\right)\right]^{k}}=\frac{\ln \left(x_{1}+\cdots+x_{k}\right)}{\ln x_{1} \cdots \cdot \ln x_{k}}, \quad x_{1}, \ldots, x_{k}>1,
$$

is the exponential-type quasi-Cauchy quotient mean $M_{f}$ generated by the logarithmic function $f=\log _{p}$, so it does not depend on $p$.

Moreover, in case $k=2$,

$$
M(x, y)=\exp \left(\frac{(\ln x)(\ln y)}{2 \ln (x+y)}\left(\sqrt{4 \frac{\ln (x+y)}{(\ln x)(\ln y)} \ln 2+1}+1\right)\right), \quad x, y>1 .
$$


Proof. Take $k \in \mathbb{N}, k \geq 2, I=(1, \infty)$, and the function $f=\log _{p}$. Consider first the case $p>1$. By (5) we have

$$
F(x)=\frac{\log _{p}(k x)}{\left(\log _{p} x\right)^{k}}=(\ln p)^{k-1} \frac{\ln (k x)}{(\ln x)^{k}}, \quad x>1,
$$

$F$ is strictly decreasing and maps $(1, \infty)$ onto $(0, \infty)$. Since the function $(8)$ :

$$
\begin{aligned}
& (1, \infty)^{k} \ni\left(x_{1}, \ldots, x_{k}\right) \longmapsto \frac{\log _{p}\left(x_{1}+\cdots+x_{k}\right)}{\log _{p} x_{1} \cdots \log _{p} x_{k}} \\
& \quad=(\ln p)^{k-1} \frac{\ln \left(x_{1}+\cdots+x_{k}\right)}{\ln \left(x_{1} \cdots x_{k}\right)} \in(0, \infty)
\end{aligned}
$$

is decreasing in each variable, (that is easy to verify), by formula (7) of Theorem 2(ii), we obtain the mean

$$
M_{f}\left(x_{1}, \ldots, x_{k}\right)=F^{-1}\left((\ln p)^{k-1} \frac{\ln \left(x_{1}+\cdots+x_{k}\right)}{\ln x_{1} \cdots \cdot \ln x_{k}}\right), \quad x_{1}, \ldots, x_{k}>1 .
$$

Since we do not have the effective form of $F^{-1}$, we get

$$
(\ln p)^{k-1} \frac{\ln \left(k M\left(x_{1}, \ldots, x_{k}\right)\right)}{\left[\ln M\left(x_{1}, \ldots, x_{k}\right)\right]^{k}}=(\ln p)^{k-1} \frac{\ln \left(x_{1}+\cdots+x_{k}\right)}{\ln x_{1} \cdots \ln x_{k}}, \quad x_{1}, \ldots, x_{k}>1,
$$

whence

$$
\frac{\ln \left(k M\left(x_{1}, \ldots, x_{k}\right)\right)}{\left[\ln M\left(x_{1}, \ldots, x_{k}\right)\right]^{k}}=\frac{\ln \left(x_{1}+\cdots+x_{k}\right)}{\ln x_{1} \cdots \cdot \ln x_{k}}, \quad x_{1}, \ldots, x_{k}>1,
$$

which, in particular, shows that $M$ is independent of the choice of $p$.

If $p \in(0,1)$ the proof is similar, so we omit it.

The result in the case $k=2$ follows from the fact that one can easily determine the effective form of $F^{-1}$.

\section{Part 6: Exponential quasi-Cauchy quotient for multiplicative functions}

Proposition 5. Let $k \in \mathbb{N}, k \geq 2$, be fixed. For every $p \in \mathbb{R}, p \neq 0$, the exponential-type quasi-Cauchy quotient mean $M_{f}:(0, \infty)^{k} \rightarrow(0, \infty)$ generated by the power function $f(x)=x^{p},(x>0)$, does not depend on $p$, and

$$
M_{f}=\mathcal{B}_{k}
$$

where $\mathcal{B}_{k}$ is the Beta-type mean.

Proof. By Theorem 2 with $I=(0, \infty)$ and $f(x)=x^{p}$ we have

$$
F(x)=\frac{k^{p}}{x^{(k-1) p}}, \quad x>0,
$$

whence

$$
F^{-1}(t)=\frac{k^{\frac{1}{k-1}}}{t^{\frac{1}{(k-1) p}}}, \quad t>0
$$


and, for all $x_{1}, \ldots, x_{k}>0$,

$$
\begin{aligned}
& M_{f}\left(x_{1}, \ldots, x_{k}\right)=\frac{k^{\frac{1}{k-1}}}{\left(\frac{\left(x_{1}+\cdots+x_{k}\right)^{p}}{\left(x_{1} \cdots x_{k}\right)^{p}}\right)^{\frac{1}{(k-1) p}}} \\
& =\left(\frac{k x_{1} \cdots \cdots x_{k}}{x_{1}+\cdots+x_{k}}\right)^{1 /(k-1)}=\mathcal{B}_{k}\left(x_{1}, \ldots, x_{k}\right) .
\end{aligned}
$$

\section{Logarithmic quasi-Cauchy quotient}

Theorem 3. Let $I \subset \mathbb{R}$ be an interval that is closed under multiplication, $f$ : $I \rightarrow(0, \infty)$ be a function; $k \in \mathbb{N}, k \geq 2$; and let $F: I \rightarrow(0, \infty)$ be given by

$$
F(x):=\frac{f\left(x^{k}\right)}{k f(x)}, \quad x \in I .
$$

Then

(i) if $F$ is one-to-one and

$$
\left\{\frac{f\left(x_{1} \cdots x_{k}\right)}{f\left(x_{1}\right)+\cdots+f\left(x_{k}\right)}: x_{1}, \ldots, x_{k} \in I\right\} \subset\{F(x): x \in I\},
$$

then the function $M_{f}: I^{k} \rightarrow(0, \infty)$ defined by

$$
M_{f}\left(x_{1}, \ldots, x_{k}\right):=F^{-1}\left(\frac{f\left(x_{1} \cdots x_{k}\right)}{f\left(x_{1}\right)+\cdots+f\left(x_{k}\right)}\right)
$$

is a correctly defined symmetric $k$-variable premean in $I$;

(ii) if $f$ is continuous and the function

$$
I^{k} \ni\left(x_{1}, \ldots x_{k}\right) \longmapsto \frac{f\left(x_{1} \cdots \cdots x_{k}\right)}{f\left(x_{1}\right)+\cdots+f\left(x_{k}\right)}
$$

is strictly increasing (decreasing) in one of the variables, then $F$ is strictly increasing (decreasing) and the function $M_{f}$ defined by (11) is a continuous symmetric strictly increasing $k$-variable mean in $I$.

Proof. Analogous as in Theorem 1.

Definition 3. Under the assumptions of Theorem 3 (ii), the function $M_{f}: I^{k} \rightarrow$ $I$ is referred to as a logarithmic-type quasi-Cauchy quotient mean generated by $f$ (or of generator $f$ ).

Problem 3. Let $f: I \rightarrow(0, \infty)$ and $g: I \rightarrow(0, \infty)$ satisfy the conditions of Theorem 3. Determine conditions under which the logarithmic-type quasiCauchy quotient premeans $M_{f}$ and $M_{g}$ are equal. 
Remark 11. Put $G(x):=\frac{g(k x)}{k g(x)}$ and $\varphi:=G \circ F^{-1}$. Now the equality $M_{f}=M_{g}$ leads to the functional equation

$$
\varphi\left(\frac{f\left(x_{1} \cdots x_{k}\right)}{f\left(x_{1}\right)+\cdots+f\left(x_{k}\right)}\right)=\frac{g\left(x_{1} \cdots x_{k}\right)}{g\left(x_{1}\right)+\cdots+g\left(x_{k}\right)}, \quad x_{1}, \ldots, x \in I,
$$

which for $k=2$ reduces to

$$
\varphi\left(\frac{f(x y)}{f(x)+f(y)}\right)=\frac{g(x y)}{g(x)+g(y)}, \quad x, y \in I,
$$

which is a special case of a more general equation

$$
\varphi(h(x, y))=\frac{g(x y)}{g(x)+g(y)}, \quad x, y \in I,
$$

with unknowns $\varphi, h$ and $g$.

\section{Part 7: Logarithmic quasi-Cauchy quotient for additive functions}

Applying Theorem 3 for continuous additive functions, we again obtain the Beta-type means. Indeed, we have

Proposition 6. Let $k \in \mathbb{N}, k \geq 2$, be fixed. For every $p>0$, the logarithmic-type quasi-Cauchy quotient mean $M_{f}:(0, \infty)^{k} \rightarrow(0, \infty)$ generated by the function $f(x)=p x,(x>0)$ does not depend on $p$, and

$$
M_{f}=\mathcal{B}_{k},
$$

where $\mathcal{B}_{k}$ is the Beta-type mean.

Proof. Take $k \in \mathbb{N}, k \geq 2, I=(0, \infty), p>0$, and the function $f(x)=p x$. By (9) we have

$$
F(x)=\frac{p x^{k}}{k p x}=\frac{1}{k} x^{k-1}, \quad x>0,
$$

so the function $F:(0, \infty) \rightarrow(0, \infty)$ is strictly increasing, and

$$
F^{-1}(t)=(k t)^{1 /(k-1)}, \quad t>0 .
$$

Since the function (12) for $f(x)=p x$ :

$$
(0, \infty)^{k} \ni x \longmapsto \frac{p\left(x_{1} \cdots x_{k}\right)}{p\left(x_{1}+\cdots+x_{k}\right)}
$$

is strictly increasing in each variable, the assumptions of Theorem 3(ii) are satisfied. Applying this result we obtain

$$
\begin{aligned}
M_{f}\left(x_{1}, \ldots, x_{k}\right) & =\left(k \frac{p\left(x_{1} \cdots x_{k}\right)}{p\left(x_{1}+\cdots+x_{k}\right)}\right)^{1 /(k-1)}=\left(\frac{k x_{1} \cdots x_{k}}{x_{1}+\cdots+x_{k}}\right)^{1 /(k-1)} \\
& =\mathcal{B}_{k}\left(x_{1}, \ldots, x_{k}\right)
\end{aligned}
$$

for all $x_{1}, \ldots, x_{k}>0$. 


\section{Part 8: Logarithmic quasi-Cauchy quotient for exponential functions}

Proposition 7. Let $k \in \mathbb{N}, k \geq 2$, be fixed. For every $p \in \mathbb{R}, p \neq 1$, there is a unique mean $M_{p}:(1, \infty)^{k} \rightarrow(1, \infty)$ such that $M_{p}=M_{f}$, where $M_{f}$ is a logarithmic-type quasi-Cauchy quotient mean generated by the exponential function $f(x)=p^{x}$; and the mean $M_{p}$ is given by the implicit equation

$$
\frac{1}{k} p^{\left[M_{p}\left(x_{1}, \ldots, x_{k}\right)\right]^{k}-M_{p}\left(x_{1}, \ldots, x_{k}\right)}=\frac{p^{x_{1} \cdots x_{k}}}{p^{x_{1}}+\cdots+p^{x_{k}}}, \quad x_{1}, \ldots, x_{k}>1 .
$$

Moreover the functions $M_{0}, M_{1}, M_{\infty}:(1, \infty)^{k} \rightarrow(1, \infty)$ defined as the pointwise limits

$$
M_{0}=\lim _{p \rightarrow 0} M_{p}, \quad M_{1}=\lim _{p \rightarrow 1} M_{p}, \quad M_{\infty}=\lim _{p \rightarrow \infty} M_{p}
$$

exist, and are $k$-variable means such that

$$
\begin{aligned}
& {\left[M_{0}\left(x_{1}, \ldots, x_{k}\right)\right]^{k}-M_{0}\left(x_{1}, \ldots, x_{k}\right)=x_{1} \cdots x_{k}-\min \left(x_{1}, \ldots, x_{k}\right),} \\
& x_{1}, \ldots, x_{k}>1 \text {; } \\
& {\left[M_{1}\left(x_{1}, \ldots, x_{k}\right)\right]^{k}-M_{1}\left(x_{1}, \ldots, x_{k}\right)=x_{1} \cdots x_{k}-\frac{x_{1}+\cdots+x_{k}}{k},} \\
& x_{1}, \ldots, x_{k}>1 \text {; } \\
& {\left[M_{\infty}\left(x_{1}, \ldots, x_{k}\right)\right]^{k}-M_{\infty}\left(x_{1}, \ldots, x_{k}\right)=x_{1} \cdots x_{k}-\max \left(x_{1}, \ldots, x_{k}\right) \text {, }} \\
& x_{1}, \ldots, x_{k}>1 \text {. }
\end{aligned}
$$

In the case $k=2$ we have

$$
M_{p}(x, y)=\left\{\begin{array}{ccc}
\frac{1}{2}\left(1+\sqrt{1+\log _{p} \frac{2 p^{x y}}{p^{x}+p^{y}}}\right) & \text { if } p \in(0, \infty) \backslash\{1\} \\
\frac{1}{2}(1+\sqrt{1+4 x y-4 \min (x, y)}) & \text { if } & p=0 \\
\frac{1}{2}\left(1+\sqrt{1+4 x y-4 \frac{x+y}{2}}\right) & \text { if } & p=1 \\
\frac{1}{2}(1+\sqrt{1+4 x y-4 \max (x, y)}) & \text { if } & p=\infty
\end{array}, x, y>1 .\right.
$$

Proof. Take $k \in \mathbb{N}, k \geq 2 ; I=(1, \infty) ; p>0, p \neq 1$; and let $f(x)=p^{x}$. Assume first that $p>1$. By (9) we have

$$
F(x)=\frac{p^{x^{k}}}{k p^{x}}=\frac{1}{k} p^{x^{k}-x}, \quad x>1,
$$

so the function $F:(1, \infty) \rightarrow\left(\frac{1}{k}, \infty\right)$ is strictly increasing. For the function (12) we have

$$
(1, \infty)^{k} \ni\left(x_{1}, \ldots, x_{k}\right) \longmapsto \frac{p^{x_{1} \cdots x_{k}}}{p^{x_{1}}+\cdots+p^{x_{k}}} \in\left(\frac{1}{k}, \infty\right)
$$

and, for all $x_{1}, \ldots, x_{k}>1$,

$$
\frac{\partial}{\partial x_{1}} \frac{p^{x_{1} \cdots x_{k}}}{p^{x_{1}}+\cdots+p^{x_{k}}}=\frac{p^{x_{1} \cdots x_{k}} x_{2} \cdots x_{k}\left(p^{x_{2}}+\cdots+p^{x_{k}}\right)}{\left(p^{x_{1}}+\cdots+p^{x_{k}}\right)^{2}}>0,
$$


so, by symmetry, it is strictly increasing in each of the variables. Now Theorem 3 (ii) implies that the function $M_{f}:(1, \infty)^{k} \rightarrow(1, \infty)$ given by

$$
M_{f}\left(x_{1}, \ldots, x_{k}\right)=F^{-1}\left(\frac{p^{x_{1} \cdots x_{k}}}{p^{x_{1}}+\cdots+p^{x_{k}}}\right), \quad x_{1}, \ldots, x_{k}>1,
$$

is the desired mean $M_{p}:=M_{f}$. Since, in general, we do not have the effective formula for $F^{-1}$, we get

$$
\frac{1}{k} p^{\left[M_{p}\left(x_{1}, \ldots, x_{k}\right)\right]^{k}-M_{p}\left(x_{1}, \ldots, x_{k}\right)}=\frac{p^{x_{1} \cdots \cdots x_{k}}}{p^{x_{1}}+\cdots+p^{x_{k}}}, \quad x_{1}, \ldots, x_{k}>1 .
$$

Hence, making use of the L'Hospital rule, for all $x_{1}, \ldots, x_{k}>1$, we get

$$
\begin{aligned}
& \lim _{p \rightarrow 1+}\left(\left[M_{p}\left(x_{1}, \ldots, x_{k}\right)\right]^{k}-M_{p}\left(x_{1}, \ldots, x_{k}\right)\right) \\
= & \lim _{p \rightarrow 1+} \log _{p} \frac{k p^{x_{1} \cdots \cdots x_{k}}}{p^{x_{1}}+\cdots+p^{x_{k}}}=\lim _{p \rightarrow 1+} \frac{\ln \frac{k e^{x_{1} \cdots \cdots x_{k} \ln p}}{e^{x_{1} \ln p+\cdots+e^{x} k \ln p}}}{\ln p} \\
= & \lim _{p \rightarrow 1+}\left[x_{1} \cdots x_{k}-\left(\frac{e^{x_{1} \ln p}}{e^{x_{1} \ln p}+\cdots+e^{x_{k} \ln p}} x_{1}+\cdots+\frac{e^{x_{k} \ln p}}{e^{x_{1} \ln p}+\cdots+e^{x_{k} \ln p}} x_{k}\right)\right] \\
= & x_{1} \cdots x_{k}- \\
& \lim _{p \rightarrow 1+}\left(\frac{x_{1}}{1+e^{\left(x_{2}-x_{1}\right) \ln p+\cdots+e^{\left(x_{k}-x_{1}\right) \ln p}}+\cdots+}\right. \\
& \left.\frac{x_{k}}{e^{\left(x_{1}-x_{k}\right) \ln p}+\cdots+e^{\left(x_{k-1}-x_{k}\right) \ln p}}\right) \\
= & x_{1} \cdots x_{k}-\frac{x_{1}+\cdots+x_{k}}{k} .
\end{aligned}
$$

Now the properties of the function $t^{k}-t$ imply that $M_{0}\left(x_{1}, \ldots, x_{k}\right)$ $=\lim _{p \rightarrow 0} M_{p}$ exists, $M_{0}$ is a mean as the limit of means, and

$$
\begin{aligned}
& {\left[M_{0}\left(x_{1}, \ldots, x_{k}\right)\right]^{k}-M_{0}\left(x_{1}, \ldots, x_{k}\right)=x_{1} \cdots x_{k}} \\
& -\frac{x_{1}+\cdots+x_{k}}{k}, \quad x_{1}, \ldots, x_{k}>1 .
\end{aligned}
$$

Similarly we get

$$
\begin{aligned}
& \lim _{p \rightarrow \infty}\left(\left[M_{p}\left(x_{1}, \ldots, x_{k}\right)\right]^{k}-M_{p}\left(x_{1}, \ldots, x_{k}\right)\right) \\
& =\lim _{p \rightarrow \infty}\left[x_{1} \cdots x_{k}-\left(\frac{e^{x_{1} \ln p}}{e^{x_{1} \ln p}+\cdots+e^{x_{k} \ln p}} x_{1}+\cdots+\frac{e^{x_{k} \ln p}}{e^{x_{1} \ln p}+\cdots+e^{x_{k} \ln p}} x_{k}\right)\right] \\
& =x_{1} \cdots x_{k}-\lim _{p \rightarrow \infty} \\
& \left(\frac{x_{1}}{1+e^{\left(x_{2}-x_{1}\right) \ln p}+\cdots+e^{\left(x_{k}-x_{1}\right) \ln p}}+\cdots+\frac{x_{k}}{e^{\left(x_{1}-x_{k}\right) \ln p}+\cdots+e^{\left(x_{k-1}-x_{k}\right) \ln p}}\right) \\
& =x_{1} \cdots x_{k}-\max \left(x_{1}, \ldots, x_{k}\right) \text {, }
\end{aligned}
$$

which proves the existence of the mean $M_{\infty}$. 
In the case $0<p<1$ the suitable considerations as well as the calculations of the limits $\lim _{p \rightarrow 0} M_{p}$ and $\lim _{p \rightarrow 1-} M_{p}$ are analogous, so we omit them.

In the case $k=2$, setting $x_{1}=x, x_{2}=y$, the implicit equality becomes

$$
\frac{1}{2} p^{\left[M_{p}(x, y)\right]^{2}-M_{p}(x, y)}=\frac{p^{x y}}{p^{x}+p^{y}}, \quad x, y>1,
$$

whence

$$
\left[M_{p}(x, y)\right]^{2}-M_{p}(x, y)=\log _{p} \frac{2 p^{x y}}{p^{x}+p^{y}}, \quad x, y>1, p \in[0, \infty],
$$

which is a quadratic equation for $M$. Solving it we get the result.

Remark 12. Since the function $F(x)=\frac{1}{k} p^{x^{k}-x}$ is not monotonic in the interval $(0,1)$, a similar result for this interval does not hold.

\section{Part 9: Logarithmic quasi-Cauchy quotient for multiplicative functions}

Applying Theorem 3 to multiplicative continuous generators we obtain an extension of Beta-type means.

Proposition 8. Let $k \in \mathbb{N}, k \geq 2$, be fixed. For every $p \in \mathbb{R}, p \neq 0$, the function $M_{p}:(0, \infty)^{k} \rightarrow(0, \infty)$ given by

$$
M_{p}\left(x_{1}, \ldots, x_{k}\right)=\left(\frac{k^{1 / p} x_{1} \cdots x_{k}}{\left(x_{1}^{p}+\cdots+x_{k}^{p}\right)^{1 / p}}\right)^{1 /(k-1)}
$$

is the logarithmic-type quasi-Cauchy quotient mean $M_{f}$ generated by the power function $f(x)=x^{p}$. Moreover the pointwise limits $M_{-\infty}, M_{0}, M_{\infty}:(0, \infty)^{k} \rightarrow$ $(0, \infty)$,

$$
M_{-\infty}=\lim _{p \rightarrow-\infty} M_{p}, \quad M_{\infty}=\lim _{t \rightarrow \infty} M_{p}, \quad M_{0}=\lim _{p \rightarrow 0} M_{p},
$$

exist and

$$
\begin{aligned}
M_{-\infty}\left(x_{1}, \ldots, x_{k}\right) & =\left(\frac{x_{1} \cdots x_{k}}{\min \left(x_{1}, \ldots, x_{k}\right)}\right)^{\frac{1}{k-1}}, \\
M_{-\infty}\left(x_{1}, \ldots, x_{k}\right) & =\left(\frac{x_{1} \cdots x_{k}}{\max \left(x_{1}, \ldots, x_{k}\right)}\right)^{\frac{1}{k-1}}, \\
M_{0}\left(x_{1}, \ldots, x_{k}\right) & =\left(x_{1} \cdots x_{k}\right)^{\frac{1}{k}}
\end{aligned}
$$

in particular, $M_{0}$ is the geometric mean.

Proof. Take $k \in \mathbb{N}, k \geq 2, I=(0, \infty), p \in \mathbb{R}, p \neq 0$, and the function $f(x)=x^{p}$. By $(9)$ we have

$$
F(x)=\frac{1}{k} x^{p(k-1)}, \quad x>0,
$$


so the function $F:(0, \infty) \rightarrow(0, \infty)$ is strictly increasing for $p>0$ and strictly decreasing for $p<0$, and

$$
F^{-1}(t)=(k t)^{1 / p(k-1)}, \quad t>0 .
$$

Since the function

$$
(0, \infty)^{k} \ni\left(x_{1}, \ldots, x_{k}\right) \longmapsto \frac{\left(x_{1} \cdots x_{k}\right)^{p}}{x_{1}^{p}+\cdots+x_{k}^{p}}
$$

is strictly increasing in each variable if $p>0$ and strictly decreasing in each variable if $p<0$, the first result follows from Theorem 3 (ii).

We omit the easy verification of the results concerning the limits.

Remark 13. Note that $M_{1}=\mathcal{B}_{k}$.

Corollary 2. Let $k=2$. Then for every $p \in \mathbb{R}$ the geometric mean $G=M_{0}$ is invariant with respect to the mean-type mapping $\left(M_{-p}, M_{p}\right):(0, \infty)^{2} \rightarrow$ $(0, \infty)^{2}$, i.e.

$$
M_{0} \circ\left(M_{-p}, M_{p}\right)=M_{0},
$$

which implies that the sequence $\left(\left(M_{-p}, M_{p}\right)^{n}: n \in \mathbb{N}\right)$ of iterates of $\left(M_{-p}, M_{p}\right)$ is pointwise convergent in $(0, \infty)^{2}$, and

$$
\lim _{n \rightarrow \infty}\left(M_{-p}, M_{p}\right)^{n}=\left(M_{0}, M_{0}\right) .
$$

Proof. Since, for all $x, y>0$,

$$
\begin{aligned}
M_{0} & \circ\left(M_{-p}, M_{p}\right)(x, y) \\
& =\sqrt{\frac{2^{1 /(-p)} x y}{\left(x^{(-p)}+y^{(-p)}\right)^{1 /(-p)}} \frac{2^{1 / p} x y}{\left(x^{p}+y^{p}\right)^{1 / p}}}=\sqrt{x y}=M_{0}(x, y),
\end{aligned}
$$

the result follows from [7].

\section{Multiplicative quasi-Cauchy quotient}

Theorem 4. Let $I \subset(0, \infty)$ be an interval that is closed under multiplication, $f: I \rightarrow(0, \infty)$ be a function; $k \in \mathbb{N}, k \geq 2$; and let $F: I \rightarrow(0, \infty)$ be given by

$$
F(x):=\frac{f\left(x^{k}\right)}{[f(x)]^{k}}, \quad x \in I .
$$

Then 
(i) if $F$ is one-to-one and

$$
\left\{\frac{f\left(x_{1} \cdots x_{k}\right)}{f\left(x_{1}\right) \cdots f\left(x_{k}\right)}: x_{1}, \ldots, x_{k} \in I\right\} \subset\{F(x): x \in I\},
$$

then the function $M_{f}: I^{k} \rightarrow(0, \infty)$ defined by

$$
M_{f}\left(x_{1}, \ldots, x_{k}\right):=F^{-1}\left(\frac{f\left(x_{1} \cdots x_{k}\right)}{f\left(x_{1}\right) \cdots f\left(x_{k}\right)}\right)
$$

is a correctly defined symmetric $k$-variable premean in $I$;

(ii) if $f$ is continuous and the function

$$
I^{k} \ni\left(x_{1}, \ldots x_{k}\right) \longmapsto \frac{f\left(x_{1} \cdots x_{k}\right)}{f\left(x_{1}\right) \cdots f\left(x_{k}\right)}
$$

is strictly increasing (decreasing) in one of the variables, then $F$ is strictly increasing (decreasing) and the function $M_{f}$ defined by (15) is a continuous symmetric strictly increasing $k$-variable mean in $I$.

Proof. Analogous as in Theorem 1.

Definition 4. Under the assumptions of Theorem 4 (ii), the function $M_{f}: I^{k} \rightarrow$ $I$ is referred to as a multiplicative-type quasi-Cauchy quotient mean generated by $f$ (or of generator $f$ ).

Problem 4. Let $f: I \rightarrow(0, \infty)$ and $g: I \rightarrow(0, \infty)$ satisfy the conditions of Theorem 4. Determine conditions under which the logarithmic-type quasiCauchy quotient premeans $M_{f}$ and $M_{g}$ are equal.

Remark 14. Put $G(x):=\frac{g(k x)}{k g(x)}$ and note that the equality $M_{f}=M_{g}$ leads to the functional equation

$$
\varphi\left(\frac{f\left(x_{1} \cdots x_{k}\right)}{f\left(x_{1}\right) \cdots f\left(x_{k}\right)}\right)=\frac{g\left(x_{1} \cdots x_{k}\right)}{g\left(x_{1}\right) \cdots g\left(x_{k}\right)}, \quad x_{1}, \ldots, x \in I,
$$

where $\varphi:=G \circ F^{-1}$ is defined on the set

$$
J_{f}:=\left\{\frac{f\left(x_{1} \cdots x_{k}\right)}{f\left(x_{1}\right) \cdots f\left(x_{k}\right)}: x_{1}, \ldots, x_{k}>1\right\} .
$$

In the case $k=2$ this equation reduces to

$$
\varphi\left(\frac{f(x y)}{f(x) f(y)}\right)=\frac{g(x y)}{g(x) g(y)}, \quad x, y \in I,
$$

which is a special case of the functional equation

$$
\varphi(h(x, y))=\frac{g(x y)}{g(x) g(y)}, \quad x, y \in I,
$$

where $\varphi, h$ and $g$ are unknown.

A partial solution of Problem 4 gives the following 
Remark 15. Assume that $f:(1, \infty) \rightarrow(0, \infty)$ is continuously differentiable and locally not a power function (i.e. it is not of the form $x \longmapsto \alpha x^{p}$ on any nonempty subinterval of $I)$, and $g:(1, \infty) \rightarrow(0, \infty)$ is continuous. Let

$$
J:=\left\{\frac{f(x y)}{f(x) f(y)}: x, y \in(1, \infty)\right\} .
$$

If $f, g$ and $\varphi: J \rightarrow(0, \infty)$ satisfy Eq. (17), then there are $a, b, c \in \mathbb{R}, a>$ $0, c>0$ such that

$$
g(x)=c x^{b}[f(x)]^{a}, \quad x \in I ; \quad \varphi(t)=\frac{t^{a}}{e^{c}}, \quad t \in J .
$$

Proof. Setting

$$
s=\log x, \quad t=\log y, \quad x, y \in I,
$$

and

$$
\Phi:=\log \circ \varphi \circ \exp , \quad F:=\log \circ f \circ \exp , \quad G:=\log \circ g \circ \exp ,
$$

it is easy to see, that Eq. (17) is equivalent to

$$
\Phi(F(s+t)-F(s)-F(t))=G(s+t)-G(s)-G(t), \quad s, t \in \log (I) .
$$

Since $\Phi$ is continuous, $F$ is differentiable, not affine on any subinterval of $\log (I)$, and $g$ is continuous, Bruce Ebanks' result [3] (see also [8], Remark 5), we conclude that there are some real numbers $a, b, C$ such that

$$
G(s)=a F(s)+b s+C, \quad s \in \log I ; \quad \text { and } \quad \Phi(u)=a u-C, \quad u \in J_{F},
$$

where

$$
J:=\{F(s+t)-F(s)-F(t): s, t \in \log (I)\} .
$$

Hence, setting $c=e^{C}$, we obtain

$$
g(x)=c x^{b}[f(x)]^{a}, \quad x \in I ; \quad \text { and } \quad \varphi(t)=\frac{t^{a}}{c}, \quad t \in J .
$$

Remark 16. In this remark the interval $(1, \infty)$ can be replaced by $(0,1)$ and $(0, \infty)$.

Remark 17. Ebanks proved his result in [3] using some regularity results of Járai [5] (see also [2], where stronger regularity is assumed).

Part 10: Multiplicative quasi-Cauchy quotient for exponential functions

Proposition 9. Let $k \in \mathbb{N}, k \geq 2$, be fixed. 
(i) Let $I=(1, \infty)$. Then for every $p>0, p \neq 1$, the exponential function $f(x)=p^{x}, x \in I$, generates a unique multiplicative-type quasi-Cauchy quotient mean $M:(1, \infty)^{k} \rightarrow(1, \infty)$; it is given by the implicit equality

$$
M^{k}-k M=x_{1} \cdots x_{k}-\left(x_{1}+\cdots+x_{k}\right), \quad x_{1}, \ldots, x_{k}>1,
$$

and it does not depend on $p$.

Moreover, in case $k=2$,

$$
M(x, y)=1+\sqrt{(x-1)(y-1)} .
$$

(ii) Let $I=(0,1)$. Then for every $p>0, p \neq 1$, the exponential function $f(x)=p^{x}, x \in I$, generates a unique multiplicative-type quasi-Cauchy quotient mean $M:(0,1)^{k} \rightarrow(0, \infty)$; it is given by the implicit equality $k M-M^{k}=x_{1}+\cdots+x_{k}-x_{1} \cdots x_{k}, \quad x_{1}, \ldots, x_{k} \in(0,1)$, and it does not depend on $p$. Moreover, in case $k=2$,

$$
M(x, y)=1-\sqrt{(1-x)(1-y)} .
$$

Proof. (ii) Take $k \in \mathbb{N}, k \geq 2, I=(1, \infty), p>0, p \neq 1$, and the function $f(x)=p^{x}$ in Theorem 4. By (13) we have

$$
F(x)=\frac{p^{x^{k}}}{\left(p^{x}\right)^{k}}=p^{x^{k}-k x}, \quad x>1 .
$$

Assume first that $p>1$. Since $F^{\prime}(x)=(k \ln p) p^{x^{k}-k x}\left(x^{k-1}-1\right)$, it follows that $F$ is strictly increasing in $(1, \infty)$ and maps this interval onto $\left(p^{1-k}, \infty\right)$. Since for all $x_{1}, \ldots, x_{k}>1$,

$$
\frac{\partial}{\partial x_{1}} \frac{p^{x_{1} \cdots x_{k}}}{p^{x_{1}} \cdots \cdots p^{x_{k}}}=(\ln p)\left(x_{2} \cdots x_{k}-1\right) p^{x_{1} \cdots \cdot x_{k}-\left(x_{1}+\cdots+x_{k}\right)}>0
$$

the function (16) with $f(x)=p^{x}$ is strictly increasing in each variable in $(1, \infty)^{k}$. By Theorem 4 (ii), there is a unique mean $M:(1, \infty)^{k} \rightarrow(1, k)$ such that

$$
p^{M^{k}-k M}=\frac{p^{x_{1} \cdots x_{k}}}{p^{x_{1}} \cdots \cdot p^{x_{k}}}=p^{x_{1} \cdots \cdot x_{k}-\left(x_{1}+\cdots+x_{k}\right)},
$$

or, equivalently, such that

$$
M^{k}-k M=x_{1} \cdots x_{k}-\left(x_{1}+\cdots+x_{k}\right),
$$

which shows that $M$ does not depend on $p$.

If $0<p<1$, then $F$ and the respective function (16) are decreasing and we argue similarly.

In the case $k=2$, setting $x=x_{1}, x_{2}=y$, solving this quadratic equation $M^{2}-2 M=x y-(x+y)$ we get

$$
M(x, y)=1+\sqrt{(x-1)(y-1)}, \quad x, y>1,
$$

which concludes the proof of (i). We omit a similar argument for (ii). 
Remark 18. For $k=2$ the mean $M$ is a "translated" geometric mean or $\varphi$ -conjugate geometric mean with $\varphi(t)=t-1$, i.e. $M(x, y)=\varphi^{-1}(G(\varphi(x)$, $\varphi(y)))$.

Remark 19. In case $k=2$ the function $M:(0, \infty)^{2} \rightarrow(0, \infty)$ defined by

$$
M(x, y):=\left\{\begin{array}{ccc}
1+\sqrt{(x-1)(y-1)} & \text { if } & (x, y) \in(1, \infty)^{2} \\
1-\sqrt{(1-x)(1-y)} \text { if } & (x, y) \in(0,1)^{2} \\
1 & \text { if } \min (x, y) \leq 1 \leq \max (x, y)
\end{array}\right.
$$

is a mean. It joins two means defined separately in the intervals $(1, \infty)$ and $(0,1)$ in Proposition 9.

\section{Part 11: Multiplicative quasi-Cauchy quotient for logarithmic functions}

Proposition 10. Let $k \in \mathbb{N}, k \geq 2$, be fixed. For every $p>0, p \neq 1$, the logarithmic function $f=\log _{p}$ generates an independent on $p$ multiplicativetype quasi-Cauchy quotient mean $M:(1, \infty)^{k} \rightarrow(1, \infty)$ given by

$$
M\left(x_{1}, \ldots, x_{k}\right)=\exp \mathcal{B}_{k}\left(\ln x_{1}, \ldots, \ln x_{k}\right) .
$$

Proof. Take $k \in \mathbb{N}, k \geq 2, I=(1, \infty), p>1$, and the function $f=\log _{p}$. By (13) we have

$$
F(x)=\frac{k \log _{p} x^{k}}{\left(\log _{p} x\right)^{k}}=\frac{k}{\left(\log _{p} x\right)^{k-1}}, \quad x>1,
$$

so the function $F:(1, \infty) \rightarrow(0, \infty)$ is strictly decreasing in $(1, \infty)$. Since the function (16)

$$
(1, \infty)^{k} \ni\left(x_{1}, \ldots, x_{k}\right) \longmapsto \frac{\log _{p}\left(x_{1} \cdots x_{k}\right)}{\left(\log _{p} x_{1}\right) \cdots\left(\log _{p} x_{k}\right)}
$$

is decreasing in each variable (we omit calculations), and

$$
F^{-1}(t)=p^{\left(\frac{k}{t}\right)^{1 /(k-1)}}, \quad t>0,
$$

making use of (15), we conclude that

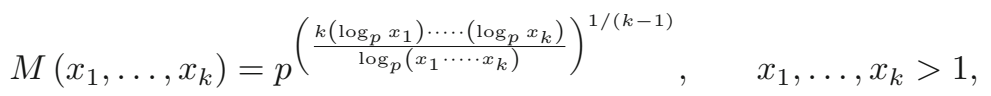

is a $k$-variable mean in $(1, \infty)$. Since $\log _{p} x=\frac{\ln x}{\ln p}$, we have, for all $x_{1}, \ldots, x_{k}>$ 1 , 


$$
\begin{aligned}
M\left(x_{1}, \ldots, x_{k}\right) & =p^{\left(\frac{k\left(\frac{\ln x_{1}}{\ln p}\right) \cdots\left(\frac{\ln x_{k}}{\ln p}\right)}{\frac{\ln \left(x_{1} \cdots \cdot x_{k}\right)}{\ln p}}\right)^{1 /(k-1)}}=\left(p^{\left.\frac{1}{\ln p}\right)\left(\frac{k\left(\ln x_{1}\right) \cdots \cdot\left(\ln x_{k}\right)}{\ln \left(x_{1} \cdots \cdots x_{k}\right)}\right)^{1 /(k-1)}}\right. \\
& =e^{\left(\frac{k\left(\ln x_{1}\right) \cdots \cdot\left(\ln x_{k}\right)}{\ln \left(x_{1} \cdots \cdots x_{k}\right)}\right)^{1 /(k-1)}}=e^{\left(\frac{k\left(\ln x_{1}\right) \cdots \cdot\left(\ln x_{k}\right)}{\ln x_{1}+\cdots+\ln x_{k}}\right)^{1 /(k-1)}} \\
& =\exp \mathcal{B}_{k}\left(\ln x_{1}, \ldots, \ln x_{k}\right)
\end{aligned}
$$

so $M$ does not depend on $p$.

We omit similar calculations in the case $p \in(0,1)$.

Remark 20. The mean $M$ is logarithmically conjugate to the mean $\mathcal{B}_{k}$. The above proposition remains true on replacing the interval $(1, \infty)$ by $(0,1)$.

\section{Part 12: Multiplicative quasi-Cauchy quotient for additive functions}

Remark 21. The additive functions do not generate any multiplicative-type quasi-Cauchy quotient means.

Indeed, taking $k \in \mathbb{N}, k \geq 2, I=(0, \infty), p>0$, and the function $f(x)=p x$, by (13) we have

$$
F(x)=\frac{p x^{k}}{(p x)^{k}}=\frac{1}{p^{k-1}}, \quad x>0,
$$

so $F$ is a constant function.

\section{Acknowledgements}

The author is grateful to anonymous referees for their careful corrections to and valuable comments on the original version of this paper.

Open Access. This article is licensed under a Creative Commons Attribution 4.0 International License, which permits use, sharing, adaptation, distribution and reproduction in any medium or format, as long as you give appropriate credit to the original author(s) and the source, provide a link to the Creative Commons licence, and indicate if changes were made. The images or other third party material in this article are included in the article's Creative Commons licence, unless indicated otherwise in a credit line to the material. If material is not included in the article's Creative Commons licence and your intended use is not permitted by statutory regulation or exceeds the permitted use, you will need to obtain permission directly from the copyright holder. To view a copy of this licence, visit http:// creativecommons.org/licenses/by/4.0/.

Publisher's Note Springer Nature remains neutral with regard to jurisdictional claims in published maps and institutional affiliations. 


\section{References}

[1] Bullen, P.S.: Handbook of Means and Their Inequalities. Kluwer, Dordrecht (2003)

[2] Ebanks, B.: Generalized Cauchy difference functional equations. Aequat. Math. 70, 154$176(2005)$

[3] Ebanks, B.: Generalized Cauchy difference equations. II. Proc. Am. Math. Soc 136(11), 3911-3919 (2008)

[4] Himmel, M., Matkowski, J.: Beta-type means. J. Differ. Equ. Appl. 24(5), 753-772 (2018)

[5] Járai, A.: Regularity Properties of Functional Equations in Several Variables. Springer, New York (2005)

[6] Kuczma, M.: Functional Equation in a Single Variable. Polish Scientific Publishers, Warszawa (1968)

[7] Matkowski, J.: Iterations of mean-type mappings and invariant means. Ann. Math. Sil. 13, 211-226 (1999)

[8] Matkowski, J.: Cauchy differences and means, to appear in Ann. Univ. Sci. Budapest. Sect. Comput

[9] Reich, L..: In Report of Meeting, the Thirty-seventh International Symposium on Functional Equations, May 16-23, 1999, Huntington. WV. Aequationes Math. 60, 175-200 (2000)

Janusz Matkowski

Faculty of Mathematics, Computer Science and Econometrics

University of Zielona Góra

Szafrana 4A

65-516 Zielona Gora

Poland

e-mail: J.Matkowski@wmie.uz.zgora.pl

Received: September 16, 2020

Revised: November 11, 2021

Accepted: November 11, 2021 